\title{
In vitro antibacterial activity of Spirulina platensis extracts against clinical isolates of Salmonella enterica serovars Typhi and Paratyphi (SUBP03)
}

\author{
Sunjukta Ahsan ${ }^{1 *}$, Md. Shamsul Arefin ${ }^{1}$, John Litol Munshi², Mst. Nadira Begum², Maisha Maliha ${ }^{1}$, \\ Sahida Rahman ${ }^{1}$, Anindita Bhowmik ${ }^{3}$ and Md. Shahidul Kabir ${ }^{4}$ \\ ${ }^{1}$ Department of Microbiology, University of Dhaka, Dhaka 1000; ${ }^{2}$ Bangladesh Council of Scientific and \\ Industrial Research, Dr. Qudrat-i-Khuda Road, Dhanmondi, Dhaka-1205; ${ }^{3}$ Department of Microbiology, \\ Jagannath University, Dhaka, Bangladesh, ${ }^{4}$ Department of Microbiology, Stamford University Bangladesh, 51, \\ Siddeswari Road, Dhaka-1217
}

Received 29 May 2015/Accepted 07 July 2015

\begin{abstract}
Natural therapy has its inherent attraction in that there are limited side-effects. Spirulina platensis, a naturally occurring algae, has been reported to have more than one therapeutic advantage. It is also used as a source of natural protein and vitamins in many parts of the world. In the present investigation, the antimicrobial activity of $S$. platensis against clinical isolates of Salmonella enterica serovar Typhi $(n=17)$ and Paratyphi $(n=3)$ was investigated. Ethanolic and chloroform extracts of $S$. platensis $(40 \mathrm{mg} / \mathrm{mL})$ were investigated for antimicrobial effect. Variable zones of inhibition were observed for the former extract whereas the latter did not show any inhibitory effect on growth of the test organism. Diameters of the zones of inhibition were 9.7-14.0 mm for $S$. Paratyphi and 9.5-16.0 mm for $S$. Typhi. The presence of zones of inhibition at the concentration of $40 \mathrm{mg} / \mathrm{ml}$ of the extract used is promising towards developing a natural remedy against infections with $S$. enterica serovars Typhi and Paratyphi.
\end{abstract}

Key words: Spirulina platensis; Algal extract; Antibacterial activity

Nature has been a source of medicinal agents for thousands of years and an impressive number of modern drugs have been isolated from natural sources, many based on their uses in traditional medicine. In the pre-industrial era the first generation of drugs was usually simple botanicals employed in more or less their crude form. Following industrial revolution, a second generation of drugs emerged based on scientific processing of the algal extract to isolate their "active principle" which was a finer form of the original crude extract (1). There has been a rising interest of researchers for natural products from plants for the discovery of new antimicrobial and antioxidant agents in the last three decades (2-5). The cyanobacteria (Blue Green Algae) are able to produce biologically active compounds (6). They are a source of inspiration for novel drug compounds that can be exploited for human health and well-being, safer or more effective than synthetically produced antimicrobial agent. Pathogen resistance to synthetic drugs and antibiotics that are already in use makes search for plants with antimicrobial activity more important, as they can substitute for synthetic antibiotics and drugs. Spirulina platensis produce a diverse range of bioactive molecules, making them a rich source of different types

${ }^{*}$ Corresponding Author: Mailing address. Dr. Sunjukta Ahsan, Department of Microbiology, University of Dhaka, Dhaka 1000, Bangladesh. Email: Sunjukta@du.ac.bd. of medicines. Spirulina platensis or its extract show therapeutic properties, such as the ability to prevent cancers, decrease blood cholesterol level, reduce nephrotoxicity of pharmaceuticals and toxic metals and provide protection against the harmful effect of radiation (7).

The aim of the present work was to study the antimicrobial activity of cell extracts of various strains of Spirulina platensis against clinical isolates of Salmonella enterica serovar Typhi ( $S$. Typhi) and $S$. enterica serovar Paratyphi. To our knowledge, this is the first report of the antimicrobial activity of $S$. platensis extract on clinical isolates of $S$. Typhi and $S$. Paratyphi.

\section{MATERIALS AND METHODS}

Bacteria. A total of 17 S. enterica serovar Typhi and 3 Paratyphi were used in this study. The bacteria used in antibacterial assays were provided by a local hospital which were isolated from patients suffering from typhoid and paratyphoid fever. Salmonella enterica serovar Typhimurium ATCC 10428 was used as control.

Preparation of extracts. The blue-green alga, S. platensis was collected from Bangladesh Council of Scientific and Industrial Research (BCSIR) as dried powder. It was kept at room temperature. Samples were then shade dried and pressed to get coarse powder. Subsequently, the powdered samples were stored in the refrigerator. Spirulina powder was resuspended in ethanol and chloform separately to prepare solutions with strengths of $40 \mathrm{mg} / \mathrm{mL}$. From each of this preparation, $20 \mu \mathrm{l}$ was used to soak each filter paper disc.

Preparation of pure culture. A loopful of each of the microorganisms was suspended in about $10 \mathrm{ml}$ of physiological saline $(0.85 \% \mathrm{NaCl})$ in a roux bottle. It was streaked on Xylose lysine deoxycholate (XLD) agar medium and incubated at $37{ }^{\circ} \mathrm{C}$ for $24 \mathrm{~h}$. Discrete colonies were selected on the basis of their colony morphology and biochemical profile. Standard biochemical tests: Kligler Iron Agar (KIA), Motility Indole Urea (MIU), citrate agar were used for identification of Salmonella spp. 
Paper-disc diffusion method. Antibacterial activity was observed by disc diffusion method. Whatmann Filter paper discs, saturated with $20 \mu \mathrm{l}$ of ethanolic extract were aseptically placed on different culture medium supplemented with the test and control organisms. Discs were fed with solvent alone to serve as control. The plates were incubated at $37{ }^{\circ} \mathrm{C}$ and observed for zone of inhibition after overnight incubation. A control assay was conducted using Ciprofloxacin discs $(5 \mu \mathrm{g})$.

\section{RESULTS}

Pure culture of $S$. enterica serovar Typhi and Paratyphi were subcultured on XLD agar and following colony morphologies were observed (Table 1).

TABLE 1. Load of various microorganisms in the pear samples

\begin{tabular}{ccc}
\hline Microorganism & Culture media & $\begin{array}{c}\text { Colony } \\
\text { characteristics }\end{array}$ \\
\hline $\begin{array}{c}\text { Salmonella } \\
\text { Typhi }\end{array}$ & $\begin{array}{c}\text { Xylose lysine } \\
\text { deoxycholate (XLD) } \\
\text { agar }\end{array}$ & Black colonies \\
$\begin{array}{c}\text { Salmonella } \\
\text { Paratyphi }\end{array}$ & $\begin{array}{c}\text { Xylose lysine } \\
\text { deoxycholate (XLD) } \\
\text { agar }\end{array}$ & Black colonies \\
\hline
\end{tabular}

Biochemical properties of the test bacteria. All the clinical isolates of Salmonella Typhi and Paratyphi were re-confirmed by a number of biochemical tests following primary confirmation at the hospital from where they were collected. In KIA, all isolates produced blackening of butt with evidence of gas formation and red color of slant. They were motile and produced indole. They lacked urease activity and were unable to use citrate as sole carbon source.

Antimicrobial activity of the ethanol extract of Spirulina platensis. Variable antimicrobial activity was exhibited by the ethanolic extract of Spirulina platensis against the test organisms. Diameters of the zone of inhibition were 9.7-14 $\mathrm{mm}$ for $S$. Paratyphi and 9.5-16 $\mathrm{mm}$ for $S$. Typhi. With reference to ciprofloxacin, no conclusion could be drawn between sensitivity to ciprofloxacin and zone diameters obtained with ethanolic extract of $S$. platensis i.e. isolates that were resistant to ciprofloxacin did not always produce small zone sizes with Spirulina extract and vice versa. Figure 1 depicts the zone sizes produced by the clinical isolates of $S$. Typhi and $S$. Paratyphi when treated with Ciprofloxacin $(5 \mu \mathrm{g})$ and ethanolic extract of $S$. platensis.

Antimicrobial activity of the chloroform extract of Spirulina platensis. The chloroform extract of $S$. platensis was found to have no effect on the test bacteria.

\section{DISCUSSION}

Spirulina platensis is one of the most important microalga showed antimicrobial activity against many pathogenic bacteria and fungi (7). Spirulina is one of the several algal genera that have attracted special attention due to their importance as human foodstuff and there in vitro or in vivo functional properties. It has been extensively cultivated to obtain a protein rich material of

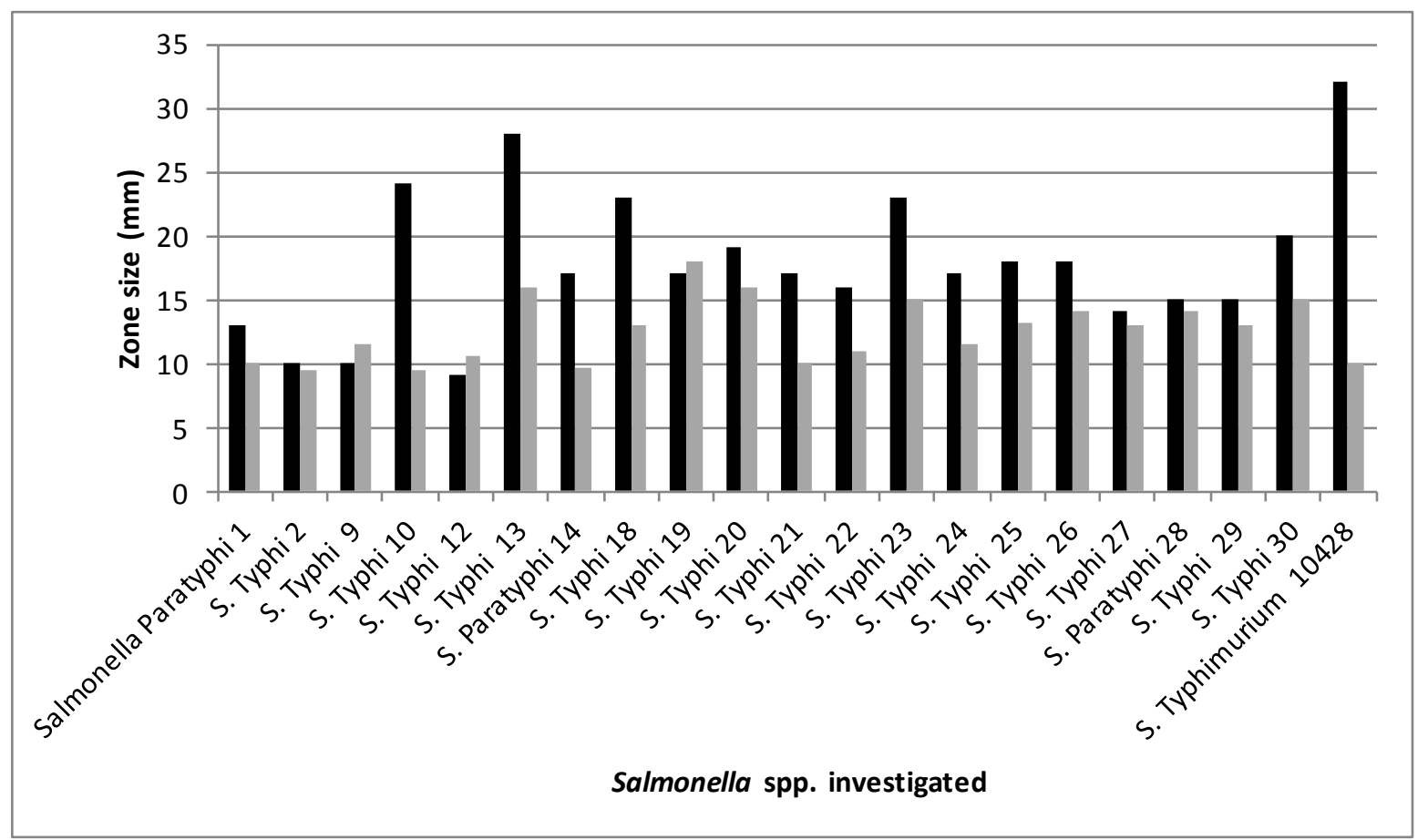

FIG. 1. Zone diameters obtained with treatment of the clinical isolates of $S$. Typhi and $S$. Paratyphi with ciprofloxacin $(5 \mu \mathrm{g})$ and $20 \mu \mathrm{crude}$ extract of Spirulina platensis. The black bars indicate ciprofloxacin treated samples and the grey bar represent zone diameters obtained when treated with ethanolic extract of S. platensis. 
nutritional or industrial use (blue pigment).

It has been recommended by medicinal experts for better health (7). In the present investigation, the antimicrobial activity of the ethanolic extract of Spirulina platensis was tested against clinical isolates of $S$. Typhi and $S$. Paratyphi. The extract was found to have variable effect on the test isolates with some isolates showing greater sensitivity to the crude ethanolic extract than others. In contrast the chloroform extract showed no antimicrobial activity against the test isolates. In earlier studies, the methanol extract was found to be more effective against Staphylococcus aureus and Salmonella typhimurium than extracts with other solvents (7). Extracts with methanol, dichloromethane, petroleum ether and ethylacetate were found to have antibacterial and antifungal (against $C$. albicans) activity against four Gram positive and six Gram negative bacteria (8). In another study (9) the ethanol extract of $S$. platensis was found to be antibacterial against $S$. aureus and E. coli and antifungal against $A$. niger and $C$. albicans.

In another study, extracts with acetone, ethanol and diethyl ether were found to exert antibacterial activity against reference strains of Klebsiella pneumoniae, Enterobacter spp., E. coli and S. typhi (10). Similar findings were reported earlier (11) using hexane, ethyl acetate, dichloromethane and methanolic extracts on $E$. coli, S. typhi, $P$. aeruginosa and $K$. pneumoniae. Both antibacterial and antifungal activities were found in the extracts with diethyl ether, acetone, ethanol and methanol against. These extracts were active against the bacteria Bacillus subtilis, Pseudomonas aeruginosa, E. coli, and $S$. aureus and against the fungi A. flavus, $F$. moniliforme, C. albicans (12). Mala et al., (13) have reported on the antibacterial activity of methanol, ethanol, propanol and aqueous extracts of $S$. platensis against K. pneumoniae, Proteus vulgaris, E. coli and $S$. aureus. $S$. aureus, E. coli and $P$. aeruginosa were also found to be inhibited by the methanolic extract of $S$. platensis (14). Other reports of the antimicrobial activity of $S$. platensis include effect against $S$. typhi (control strain), Shigella frexneri, E. coli and $C$. albicans by ethanolic extract (15), by methanolic extract against against A. flavus (16), by hexane, ethyl acetate, ethanol, butanol, acetone, methanol, chloroform extracts against $S$. epidermis, $A$. liquefaciens and C. glabrata (17), by ethanol extract against $S$. aureus, E. coli, $P$. aeruginosa, Klebsiella sp. (18), by methanol extract against $B$. subtilis, E. coli, $P$. vulgaris and $C$. albicans (19), by ethanol and aqueous extracts against the bacteria Vibrio alginolyticus, Pseudomonas fluorescens, P. aeruginosa, Aeromonas hydrophila and $A$. salmonicida and the fungi Aspergillus niger, Penicillium javanicum, Candida albicans and Trichoderma viride (20), by methanol, ethanol and aqueous extracts against $P$. fluorescence, $P$. aeruginosa, $P$. putida, $V$. alginolyticus, Vibrio flavalis, a $V$. fisheri and E. coli (21).

The present study differs from previous studies in that it was conducted on clinical isolates of $S$. Typhi and $S$. Paratyphi. The sensitivity of the isolates was compared to ciprofloxacin as a control assay. There was no correlation between the sensitivity of the isolates to the antibiotic and the zone diameters obtained with the ethanolic extract of $S$. platensis. Moreover, the sizes of the zone of inhibition were not comparable to that obtained for ciprofloxacin. At $40 \mathrm{mg} / \mathrm{ml}$ concentration, the ethanolic extract generated zones of inhibition significantly smaller than that obtained with ciprofloxacin. Future studies need to be carried out with increased concentration of the extract to obtain increased zones of inhibition. Other solvents like methanol, essential oil, water may be used for extraction of chemical ingredients imparting antimicrobial activity from $S$. platensis. Active ingredients may be separated and tested for designing new generation antibiotics. However, the finding from the present investigation is highly promising in view of the fact that the ethanolic extract was successful to inhibit the growth of the clinical isolates of the tested bacteria. Further research carried out along this line may prove to be fruitful in bringing out a natural solution against infection with $S$. Typhi and $S$. Paratyphi.

\section{REFERENCES}

1. Kumar V, Tirumalai PS, Singh A, Bhatnagar AK, Shrivastava JN 2013. Natural Compounds from Algae and Spirulina platensis \& its Antimicrobial Activity. Indo Global J. Pharma. Sci. 3 (3): 212-223.

2. Ahmer BMM, Tran M, Heffron F. 1999. The virulence plasmid of Salmonella typhimurium is self-transmissible. J. Bacteriol. 181: 1364-1368.

3. Bardwell JCA. 1994. Building bridges: disulphide bond formation in the cell. Mol. Microbiol. 14: 199-205.

4. Baumler AJ, Gilde AJ, Tsolis RM, van der Velden AWM, Ahmer BMM, Heffron F. 1997. Contribution of horizontal gene transfer and deletion events to development of distinctive patterns of fimbrial operons during evolution of Salmonella serovars. J. Bacteriol. 179: 317-322

5. Baumler AJ, Tsolis RM, Bowe FA, Kusters JG, Hoffmann S, Heffron F. 1996. The pef fimbrial operon of Salmonella typhimurium mediates adhesion to murine small intestine and is necessary for fluid accumulation in the infant mouse. Infect. Immun. 64: 61-68.

6. Berry PJ, Gantar M, Perez M, Berry G, Noriega FG. 2008. Cyanobacterial toxins as allelochemicals with potential applications as algaecides, herbicides and insecticides. Marine Drugs. 6 (2): 117-146.

7. Kumar V, Bhatnagar AK, Srivastava JN. 2011. Antibacterial activity of crude extracts of Spirulina platensis and its structural elucidation of bioactive compound. J. Med. Plants. Res. 5 (32): 7043-7048.

8. Ozdemir G, Karabay NU, Dalay MC, Pazarbasi B. 2004. Antibacterial activity of volatile extracts of Spirulina platensis. Phytother. Res. 18 (9): 754757.

9. Santoyo S, Herrero M, Javier F, Cifuentes A, Ibanez E, Jaime L. 2006. Functional characterization of pressurized liquid extracts of Spirulina platensis. Eur. Food Res. Technol. 224: 75-81.

10. Kulandaivel S, Prakash R, Anitha R, and Arunnagendran N. 2007. Antibacterial activity of Spirulina platensis and Oscillatoria sp. J. Plant and Appl. Microbiol. 1 (1): 127-129.

11. Kaushik P, Chauhan A. 2008. In vitro antibacterial activity of laboratory grown culture of Spirulina platensis. Ind. J. Microbiol. 48: 348-352.

12. Rania MAA, Taha HM. 2008. Antibacterial and Antifungal Activity of Cyanobacteria and Green Microalgae. Evaluation of Medium Components by Placket-Burman Design for Antimicrobial Activity of Spirulina platensis. Global J. Biotechnol. Biochem. 3 (1): 22-31. 
13. Mala R, Sarijini M, Saravanababu S, Umadevi G. 2009. Screening for antimicrobial activity of crude extracts of Spirulina platensis. J. Cell Tissue Res. 93 (3): 1951-1955.

14. Parisi AS, Younes S, Reinehr CO, Colla LM. 2009. Evaluation of antibacterial activity of S. platensis. J. Basic Appl. Pharmaceut. Sci. 30 (9): 297-301.

15. Uyisenga JP, Nzayino $P$, Seneza $R$, Hishamunda $L$, Uwantege $K$, Gasana N., et al. 2010. In Vitro Study of Antibacterial and Antifungal Activity of Spirulina platensis. Int. J. Ecol. Develop. 16 (10): 315-719.

16. Souza MM, Prietto L, Souza AC, Ribeiro AC, Badiale- Furlong E. 2011. Assessment of the antifungal activity of Spirulina platensis phenolic extract against Aspergillus flavus. Ciênc. Agrotec. 35 (6): 1050-1058.

17. Sivakumar J, Santhanan P. 2011. Antipathogenic activity of Spirulina platensis. Recent Res. Sci. Technol. 3 (4): 158-161.
18. Sudha SS, Karthic R, Rengaramunjan J, Athulya. 2011. Antimicrobial activity of Spirulina platensis and Aphanothece sp. on selected clinical bacterial isolates and its antioxidant activity. South As. J. Biol. Sci. 1: 87-98.

19. Medina-Jaritz NB, Perez-Solis DR, Ruiloba de Leon F, Olvera-Ramirez R. 2011. Antimicrobial activity of aqueous and Methanolic extracts from Arthrospira maxima. Formatex. A. Méndez-Vilas: 1267-1271.

20. Ramamurthy V, Raveendran S. 2009. Antibacterial and antifungal activity of Spirulina platensis and Lyngbya majuscule. J. Ecobiol. 24 (1): 47 - 52.

21. Pradhan J, Das BK, Sahu S, Marhual NM, Swain AK, Mishra BK, et al. 2012. Traditional antibacterial activity of freshwater microalga Spirulina platensis to aquatic pathogens. Aqua. Res. 43 (9): 1287-1295. 